\title{
Coulisses
}

Revue de théâtre

25 | Hiver 2002

Varia

\section{Le théâtre chilien au vingtième siècle}

\section{Carole Michel}

Traducteur : Coulisses

\section{CpenEdition}

Journals

Édition électronique

URL : http://journals.openedition.org/coulisses/6012

DOI : $10.4000 /$ coulisses.6012

ISSN : 2546-9460

Éditeur

Presses universitaires de Franche-Comté

Édition imprimée

Date de publication : 1 janvier 2002

Pagination : 89-99

ISBN : 2-84627-052-X

ISSN : 1150-594X

\section{Référence électronique}

Carole Michel, «Le théâtre chilien au vingtième siècle », Coulisses [En ligne], 25 | Hiver 2002, mis en ligne le 24 octobre 2019, consulté le 15 novembre 2019. URL : http://journals.openedition.org/ coulisses/6012 ; DOI : 10.4000/coulisses.6012

Ce document a été généré automatiquement le 15 novembre 2019.

Coulisses 


\section{Le théâtre chilien au vingtième siècle}

\section{Carole Michel}

Traduction : Coulisses

1 Le théâtre chilien prend son ampleur durant le $\mathrm{XX}^{\mathrm{e}}$ siècle.

Il est fort difficile de parler de "théâtre chilien» auparavant car la production nationale est bien faible et de piètre qualité. Les œuvres représentées proviennent surtout de pays étrangers, tels la France ou l'Espagne. Le théâtre français eut d'ailleurs un succès non négligeable au Chili, et Molière, par exemple y fut beaucoup joué.

2 À partir des années 30, le panorama culturel commence à évoluer, sous l'effet de plusieurs événements qui vont agir sur la réalité chilienne, et auront une incidence sur les aspects culturels et théâtraux.

3 D'abord, la guerre civile espagnole provoque la formation de groupes chez les intellectuels et la jeunesse universitaire qui désirent un changement dans la marche du pays et dans l'orientation de la culture, afin de la mettre à la portée de toutes les classes sociales du pays.

4 Le mouvement du Front Populaire, représentatif des classes moyennes et populaires, joue également un rôle important dans le domaine culturel. Ce dernier triomphe aux élections de 1938 et amène Pedro Aguirre Cerda à la présidence. Les intérêts du parti radical, qui occupe une place de choix parmi les élus, coïncide avec ceux d'une classe moyenne formée d'un grand nombre d'universitaires et d'intellectuels. La culture est un point important du programme politique, et le succès du Front Populaire est extrêmement positif pour son développement.

5 Le gouvernement tente de protéger le théâtre national agonisant en créant les Théâtres Mobiles dans tout le pays, et ce dès 1939. Le but est de réaliser une campagne de diffusion culturelle parmi la masse populaire. Grâce à cette initiative, la scène locale peut reprendre un peu de vigueur.

6 Enfin, la visite au Chili de la compagnie théâtrale de Margarita Xirgú, actrice catalane, aide l'art dramatique à sortir de sa crise. Elle amène avec elle un répertoire constitué 
essentiellement d'œuvres de Federico García Lorca, mais également de nouvelles techniques théâtrales (apparition d'un vrai décor au lieu de simples morceaux de papiers peints, utilisation de projecteurs pour remplacer les bougies et chandeliers, utilisation des jeux de lumière, de la musique, renouveau dans le domaine du costume...).

Un nouveau mouvement théâtral peut apparaître au Chili. Il aura une grande importance, car il va transformer de manière décisive la trajectoire du théâtre national.

71941 est sans doute l'année la plus importante pour le théâtre chilien du XX ${ }^{\mathrm{e}}$ siècle. Il existe à cette époque, dans quelques universités chiliennes, des groupes constitués par de jeunes étudiants inexpérimentés mais qui ont pour ambition de faire un théâtre différent. Autour de Pedro de la Barra, ils forment en février 1941 le Théâtre expérimental de l'université du Chili, TEUCH, et tentent de créer une expression scénique qui réponde aux nouveaux besoins.

8 Ils mettent alors au point un programme en quatre temps comprenant la formation d'une école de théâtre, la création d'un public théâtral, la diffusion de pièces classiques et modernes, la présentation de nouvelles valeurs. L'Université met à disposition des étudiants quelques salles afin qu'ils puissent mener à terme leurs premières mises en scène, qu'ils veulent «modernes ». Ils profitent de la longue expérience européenne en matière de mise en scène et utilisent alors les jeux de lumière, la musique, les effets auditifs...

Le Théâtre expérimental donne sa première représentation le 22 juin 1941, au « Teatro Imperio ", de Santiago. Deux pièces brèves se trouvent au programme: La guarda cuidadosa de Cervantes et Ligazón de Valle-Inclán, respectivement mises en scène par Pedro de la Barra et José Ricardo Morales.

Le but du Théâtre expérimental est d'utiliser la scène pour permettre la diffusion de la culture. Jusqu'en 1950 environ, ce sont surtout des œuvres classiques qui sont représentées, comme celles de Calderón, Cervantes, Moratín, Lope de Vega, ou García Lorca. L'Expérimental ne se limite pas au théâtre espagnol et met en scène le théâtre de Molière, Pirandello, Ibsen, Brecht ou Shakespeare pour n'en citer que quelques-uns.

11 Le succès est au rendez-vous, ce qui incite les jeunes comédiens à poursuivre leur entreprise, même s'ils rencontrent de sérieuses difficultés : il faut aller à la recherche d'un public, vendre les entrées, se charger de la confection des décors ou encore trouver des possibilités de diffusion.

Les débuts ne sont donc pas faciles, mais la ténacité de Pedro de la Barra porte ses fruits et en 1949, une École de théâtre, dépendante de l'Université existe réellement. L'enseignement de diverses disciplines relatives au théâtre y est dispensé.

Puis une commission syndicale se forme au sein du Théâtre expérimental. Elle a pour mission d'offrir des spectacles à des secteurs sociaux qui n'ont pas accès aux théâtres de la capitale : les prisons, les hôpitaux, les étudiants, les quartiers populaires...

Plus tard, ce Théâtre expérimental deviendra l'Institut de théâtre de l'université du Chili, ITUCH.

Le résultat de ces efforts, ce fut la création de festivals, de rencontres entre dramaturges, la fondation d'autres théâtres universitaires et l'édition d'ouvrages et de recherches sur le théâtre. De plus, depuis 1945, l'ITUCH organise le Concours annuel des œuvres théâtrales.

Rapidement, le succès du Théatre expérimental inonde les autres universités. En 1943, le Teatro de ensayo de la Universidad Católica (Théâtre d'essai de l'Université 
Catholique), TEUC, est créé.

Traditionnellement, le 12 octobre 1943 est pris comme la date de fondation du Théâtre d'essai, avec la présentation de El Peregrino de Josef de Valdivieso.

Les principaux fondateurs se nomment Pedro Mortheiru, Teodoro Lowey, Gabriela Roepke et Fernando Debesa, selon lequel :

La fondation du Théâtre d'essai ne fut pas un caprice de dilettantes... [...] Le Théâtre expérimental de l'université du Chili fut créé en 1941. Cette même année, apparut l'École de danse avec Ernst Uthoff, et en 1943 notre Théâtre d'essai. Ces événements ne sont pas le produit du hasard. Il s'agit de la maturation lente d'un pays vers la culture. Les personnes concrètes qui firent cela ont une importance occasionnelle. Ils devaient le faire, ni plus, ni moins, et ensuite ils s'en allèrent, et d'autres suivirent. ${ }^{1}$

13 Au départ, ils se heurtent à quelques difficultés. L'Université ne les appuie pas comme elle le devrait :

Don Carlos Casanueva [le recteur de l'université] commença à nous appuyer, mais très modérément. C'était un homme qui restait endormi quand nous allions lui parler. Mais nous le soupçonnions de faire l'idiot, car généralement nous allions lui demander de l'argent. [...] Ce fut difficile d'imposer le Théâtre à la Catholique. [...] Nous avons dû nous défendre de gens très influents. ${ }^{2}$

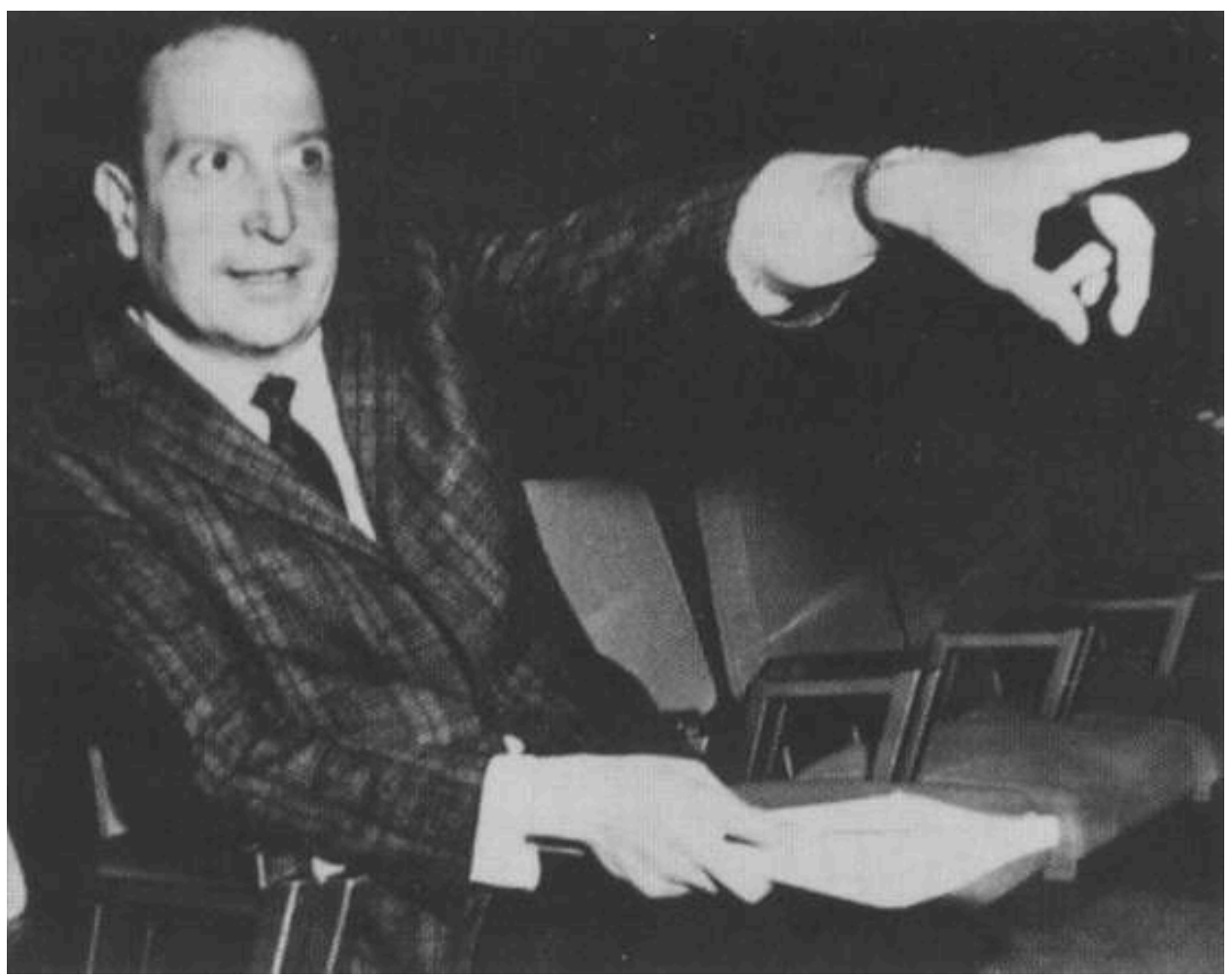

Cliché Zegers

Mais ni le scepticisme de certains, ni les problèmes économiques ne réussissent à décourager le groupe fondateur, qui représenta ses œuvres au moins une fois par an. En 1944 est mis en scène El Abanico de Goldoni, sous la direction de Pedro Mortheiru. Le succès est sans réserve, les critiques excellentes. Le Théâtre d'essai assoit sa nouvelle position. Pour Debesa,

La naissance des théâtres universitaires au Chili ne fut ni une transformation ni une prolongation. Ce fut une rupture, une guillotine. Je crois qu'une génération doit nier un peu la précédente pour pouvoir affirmer son identité. Dire "tout ceci est une 
porcherie, nous allons faire quelque chose de plus authentique, de plus vrai... c'est une façon de s'affirmer."3 jusqu'aux couches populaires et donner des représentations aux nécessiteux. La troupe se rend alors dans les prisons ou dans les bidonvilles.

Avec les années, le TEUC se consolide et peut être comparé au Théâtre de l'université $\mathrm{du}$ Chili, car il possède sa propre salle et sa propre école.

À partir de 1958, le TEUC met l'accent sur le théâtre chilien et plusieurs dramaturges, qui, plus tard, feront partie de la génération de 1950, vont naître en son sein. On peut citer entre autres Luis Alberto Heiremans, Sergio Vodanovic, Gabriela Roepke, Isidora Aguirre, David Benavente...

Santiago est devenue un centre important pour le théâtre, et plusieurs œuvres chiliennes sont présentées, aux côtés de pièces étrangères.

Le mouvement théâtral s'amplifie. De petites compagnies autonomes, indépendantes se forment ; elles complètent ainsi les théâtres officiels. Entre 1968 et 1972, un processus de rénovation du théâtre s'enclenche. La génération de 1950 connaît un vif succès et quelques-uns de ses dramaturges se tournent vers la création collective. Voient également le jour des œuvres témoignant des conditions de vie et de la lutte des secteurs populaires. Citons entre autres Pan caliente de María Asunción Requena et Los que van quedando en el camino de Isidora Aguirre, écrite en collaboration avec Manuel Rojas. 


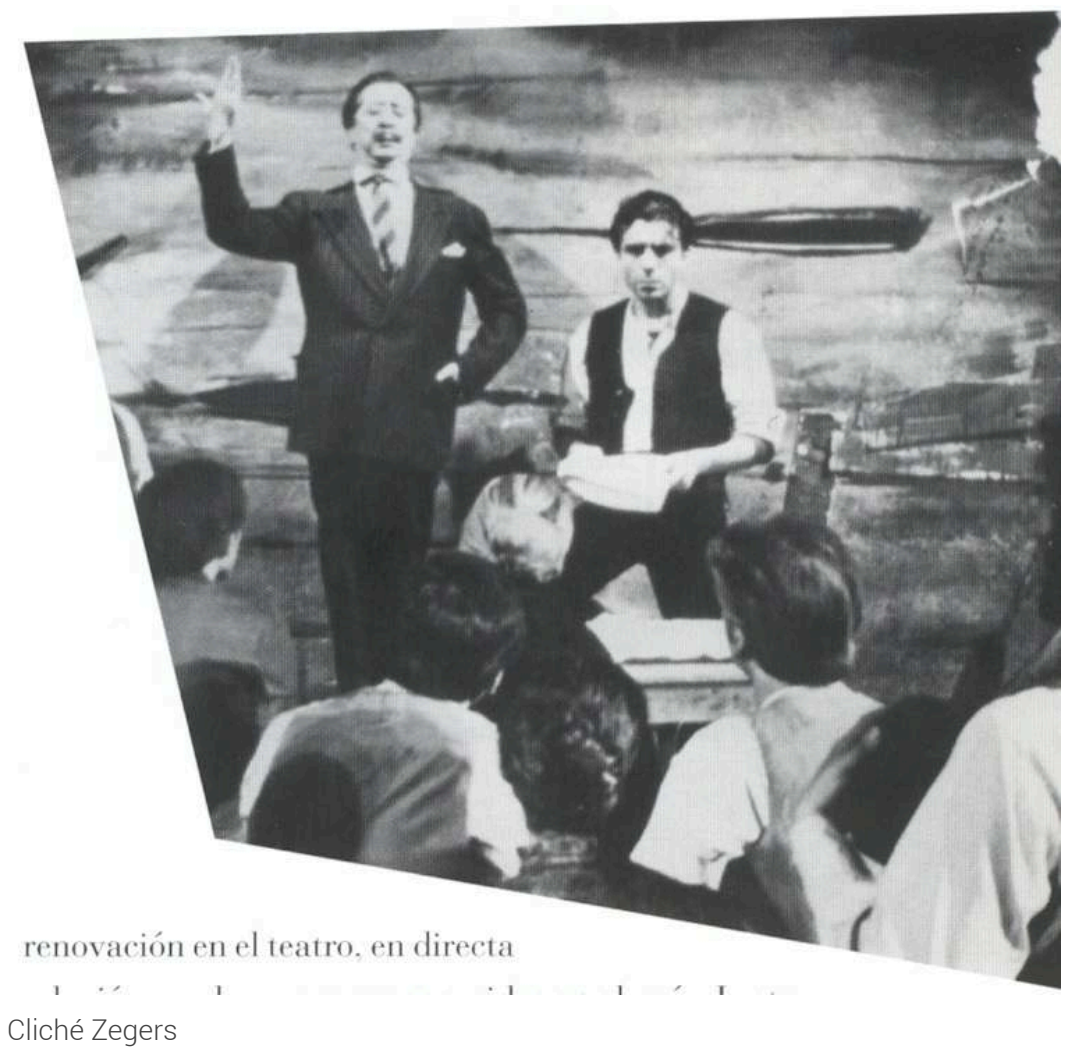

19 La création collective prend de l'ampleur. Cela suppose un renouvellement au niveau formel : le sketch devient la forme privilégiée et chacun d'entre eux évoque un aspect particulier de la vie. Au niveau de la mise en scène, on joue beaucoup sur les perceptions du spectateur grâce à l'expression corporelle, la musique, les jeux de son et de lumière. Il s'agit d'un théâtre pauvre, dépouillé de tout élément décoratif. Le corps de l'acteur se veut le moyen expressif fondamental. L'humour caustique, acerbe est de mise, et se trouve mêlé à des moments de forte émotion. Le déroulement du spectacle se base sur la rupture permanente du quatrième mur, rendant le spectateur très actif. Ce sont surtout les théâtres professionnels qui développent cette forme de dramaturgie. Parmi les plus importants, nous pouvons relever les Ateliers de création théâtrale, faisant partie du Théâtre de l'Université Catholique, ainsi que les Ateliers d'expérimentation théâtrale, appartenant à l'École des arts de la communication.

En 1968, par exemple, les Ateliers d'expérimentation théâtrale créent Peligros a 50 metros, avec la participation de Alejandro Sieveking. Il s'agit d'une œuvre évoquant la façon dont un groupe de jeunes perçoit son pays et le monde. Le but est de sensibiliser les spectateurs à la violence, l'autoritarisme, le manque de communication, la lutte des classes, etc. L'œuvre se voulait si flexible qu'il était possible d'insérer des tableaux en rapport avec les événements se déroulant au moment de la représentation.

La même année, Ictus monte Un elefante y otras zoologías de Jorge Díaz, évoquant la répression politique et les dictatures militaires en Amérique Latine.

Un an plus tard, Cuestionamos la cuestión, mise en scène par le même théâtre, questionne les institutions sociales en vigueur, telles le système politique bien sûr, mais aussi le mariage ou l'amitié. 


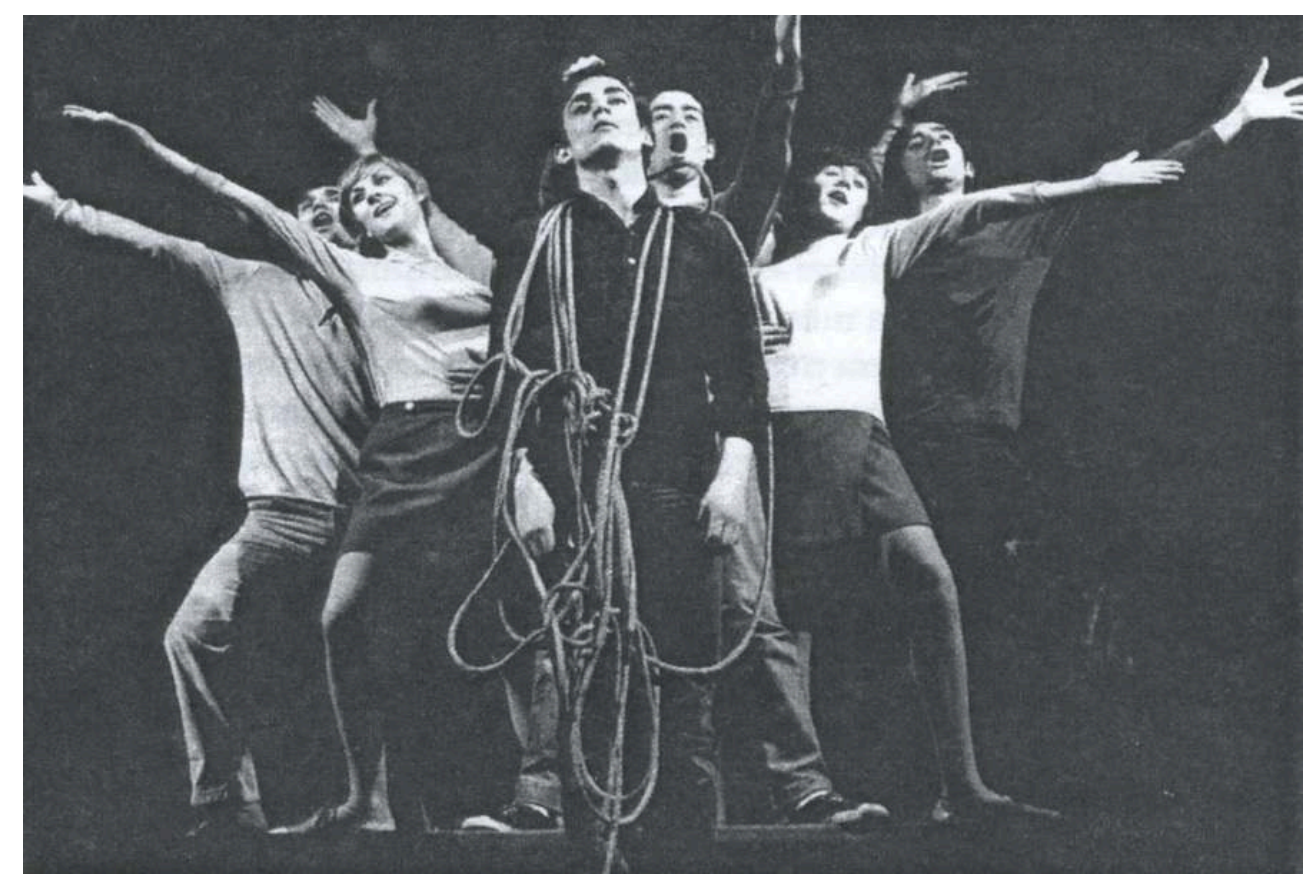

Cliché Navarro

21 Vers 1972-73, une crise s'amorce, et le coup d'État du 11 septembre 1973 n'améliore guère la situation. Entre 1973 et 1976, le milieu théâtral se désarticule; les grands projets manquent. Les théâtres universitaires se voient durement affectés par le nouveau régime. Le seul à maintenir une certaine activité est le Théâtre de l'Université Catholique. Un nouveau noyau de metteurs en scène et d'acteurs, mené par Eugenio Dittborn, parvient à garder le cap, même si en 1976, l'École des arts de la communication est fermée. Le théâtre est la seule discipline à survivre. Mais il faut préciser que les œuvres nationales ne sont plus à l'honneur. Un théâtre classique, espagnol et français, occupe de nouveau le devant de la scène :

Pendant les années qui suivirent, dans un contexte de restructurations administratives profondes et de mesures de réduction de personnel persistantes, la seule politique théâtrale possible semblait être d'assurer la subsistance de l'institution.

Les autres théâtres universitaires résistent comme ils le peuvent. Le Théâtre de l'université du Chili à Santiago doit faire face à un manque aigu de professeurs et d'élèves, ainsi qu'à la censure du gouvernement pour plusieurs mises en scène. En apparence, ce théâtre maintient sa politique de représentation : une œuvre étrangère, un classique, une pièce chilienne, mais dans ce dernier cas, toute allusion à la réalité nationale est évitée.

D'autres théâtres ferment. C'est le cas de TEKNOS, le Théâtre de l'Université Technique de Santiago. En 1976, il cesse d'exister après quinze années de fonctionnement ininterrompu. De même, les théâtres de l'université de Concepcíon, de l'Université Australe, et de l'université du Nord ferment leurs portes.

En 1974, l'Université Catholique, en collaboration avec le ministère de l'Éducation, réalise huit œuvres de théâtre télévisuelles. Mais ces dernières ne seront jamais diffusées car bon nombre d'acteurs figurent sur les fameuses "Listes Noires", interdisant l'apparition de ces personnes sur un écran de télévision. La censure fait son œuvre et près d'un quart des gens de théâtre quittent le pays. 
Seuls les théâtres indépendants semblent tirer leur épingle du jeu. De nouvelles compagnies voient le jour, et l'on compte une trentaine de groupes fin 1973. La situation se maintient jusqu'à la fin des années 70 .

Il est toutefois nécessaire de nuancer. En 1976, seuls deux théâtres déjà existant continuent leur activité : le théâtre Ictus, et le théâtre Lucho Córdoba. Toutes les autres compagnies fonctionnent de façon discontinue ou disparaissent. Quelques-unes partent à l'étranger telles la Compañia de los Cuatro et Angel. En revanche, on voit apparaître des groupes se formant de façon très éphémère, parfois le temps d'une représentation.

À partir de 1976, on assiste à une période de rénovation du théâtre. Surgit un théâtre à grand spectacle, inspiré des shows à l'américaine. Les costumes sont somptueux, les effets spéciaux largement utilisés. Parmi les productions les plus marquantes, on peut citer Fausto Shock (Compagnie Tomás Vidiella), El diluvio que viene et El violonista en el tejado (Compagnie José Aravena). Entre 1977 et 1979, ce fut un immense succès, avec plus de 100000 spectateurs. Cependant, ce type de dramaturgie tomba vite dans l'oubli. En 1978, un retour vers le théâtre national est amorcé. La saison s'ouvre avec Espejismos d'Egon Wolff. La même année est créé le Théâtre Itinérant (Teatro Itinerante), destiné à parcourir le pays. Son premier répertoire se compose de Romeo y Julieta (version de Neruda), Chañarcillo de A. Hernández et Peer Gynt.

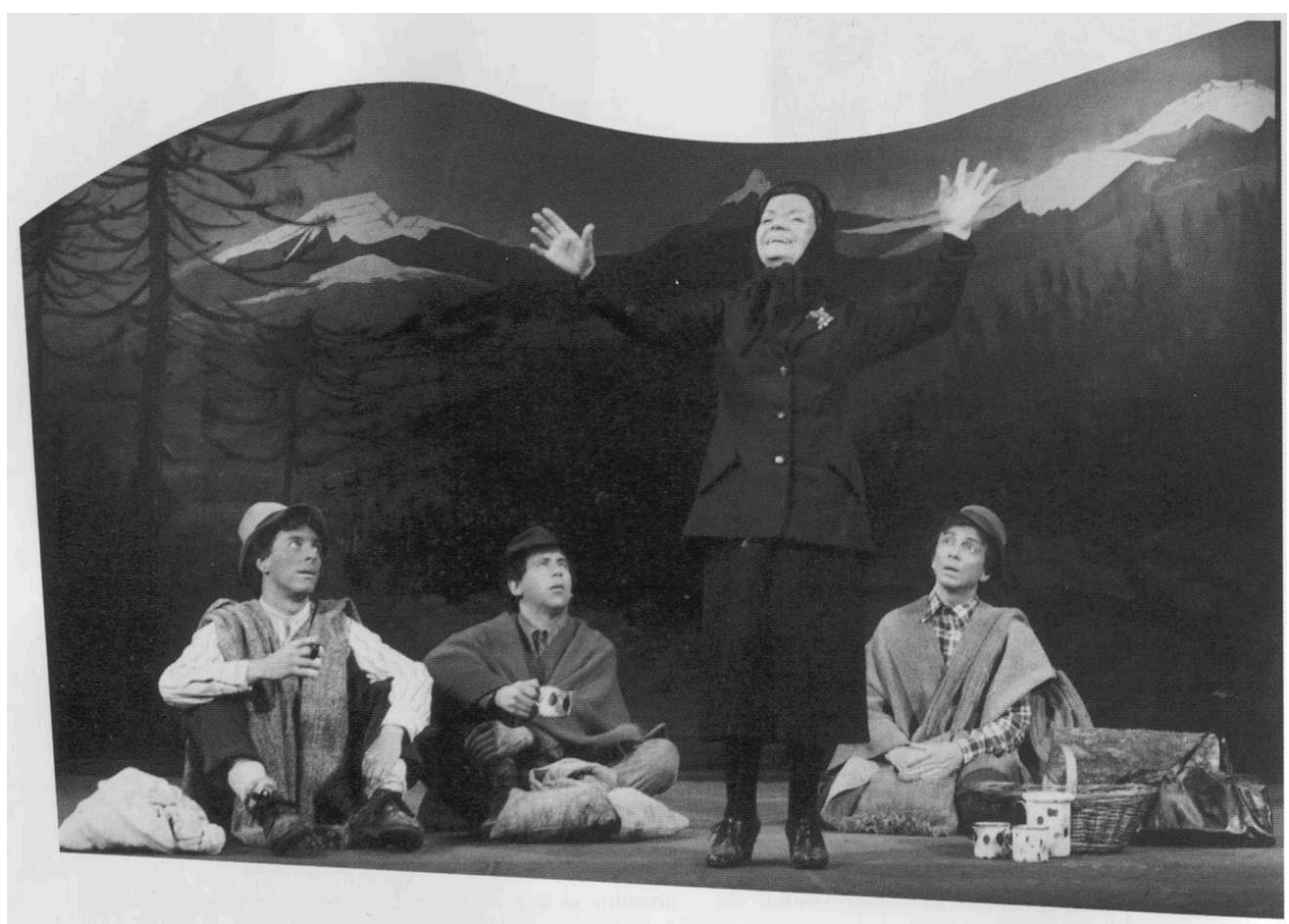

Cliché Zegers

De plus, le ministère de l'Éducation prend des mesures pour que les élèves des lycées assistent à des représentations théâtrales.

Cependant, il serait faux de dire que la censure a disparu. En 1978, parallèlement à Espejismos, le Théâtre de l'Université Catholique monte Lo crudo, lo cocido, lo podrido de Marco Antonio de la Parra. Cette pièce est censurée, jugée trop vulgaire, et l'on doit revenir à un théâtre plus classique. L'œuvre fut ensuite représentée par le Teatro Imagen, ce qui atteste de la plus grande liberté d'expression des théâtres indépendants. 
C'est justement le théâtre indépendant qui donne l'impulsion à un nouveau mouvement national. Entre 1976 et 1981, sont créées une cinquantaine d'œuvres évoquant la situation du pays.

Les groupes à l'origine de ce mouvement se nomment Ictus, Teatro Imagen, El Taller de investigación teatral... Il s'agit là de groupes solides, bien implantés, pouvant prendre des risques politiques ou économiques.

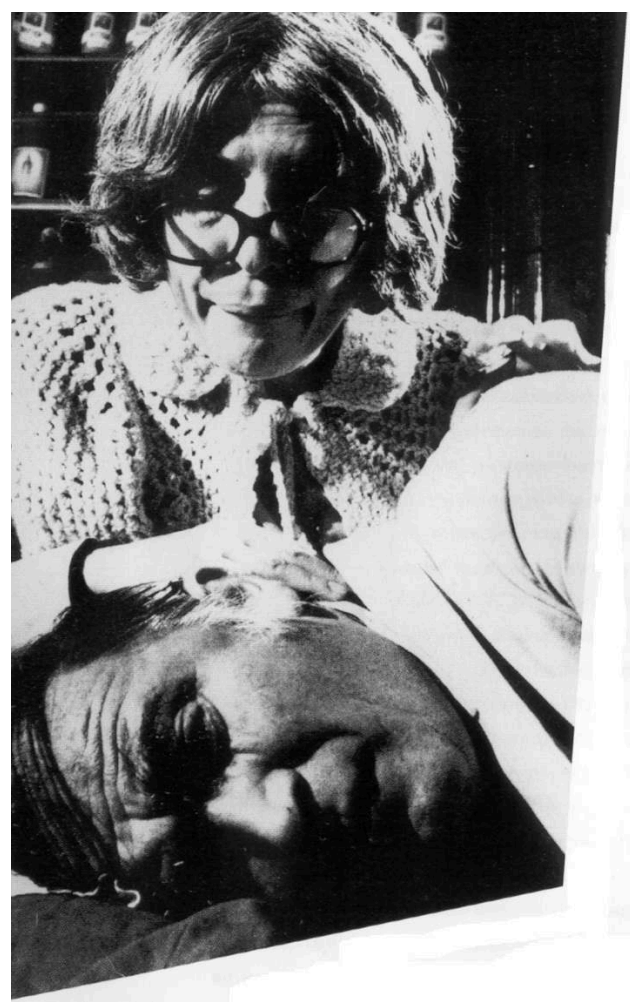

Cliché Zegers

Au début des années 80 , le pays entre en crise. La dette externe, énorme, est l'un des plus graves problèmes politiques, et le gouvernement est alors obligé de se montrer plus ouvert.

Cette ouverture est symbolisée par l'abolition de la censure concernant la publication de livres. Cela entraîne une plus grande liberté d'expression, même si des mesures sporadiques de censure interviennent encore pendant les années suivantes.

Le théâtre peut alors se permettre de traiter directement des thèmes tabous, tels la répression ou l'exil. Plusieurs groupes reviennent au pays. À cette époque, les différences entre théâtres indépendants et théâtres universitaires s'atténuent, car ces derniers ont désormais la possibilité de programmer des œuvres touchant à la réalité nationale. En 1984, le Théâtre de l'Université Catholique met en scène La balsa de la Medusa ${ }^{6}$ de Egon Wolff, critique aiguë de la bourgeoisie, et El pueblo del mal amor de Juan Radrigán.

La période est également propice à l'apparition de jeunes auteurs, assurant ainsi la relève de la génération de 1950, même si cette dernière est loin d'être éteinte. Nous pensons entre autres à Ramón Griffero.

Le théâtre connaît un second souffle. Entre 1984 et 1989, environ 170 œuvres d'auteurs nationaux sont représentées : 
Les dramaturges chiliens déjà consacrés continuaient à produire et à récolter les succès. Egon Wolff, Marco Antonio de la Parra, Jorge Díaz, Juan Radrigán, Andrés Pérez et Ramón Griffero se consolident, chacun dans son style. De nouveaux groupes, de nouveaux auteurs complètent le large éventail théâtral, tant national qu'étranger. $^{7}$

Le théâtre itinérant poursuit sa mission, présentant au pays toute une gamme d'auteurs.

L'exil et l'oppression sont évoqués dans des œuvres comme Regreso sin causa de Jaime Miranda ou Ligeros de equipaje de Jorge Díaz.

Parmi les auteurs les plus représentés lors de cette période, on trouve Luis Rivano, Alejandro Sieveking, Fernando Josseau, Fernando Cuadra, Jaime Miranda.

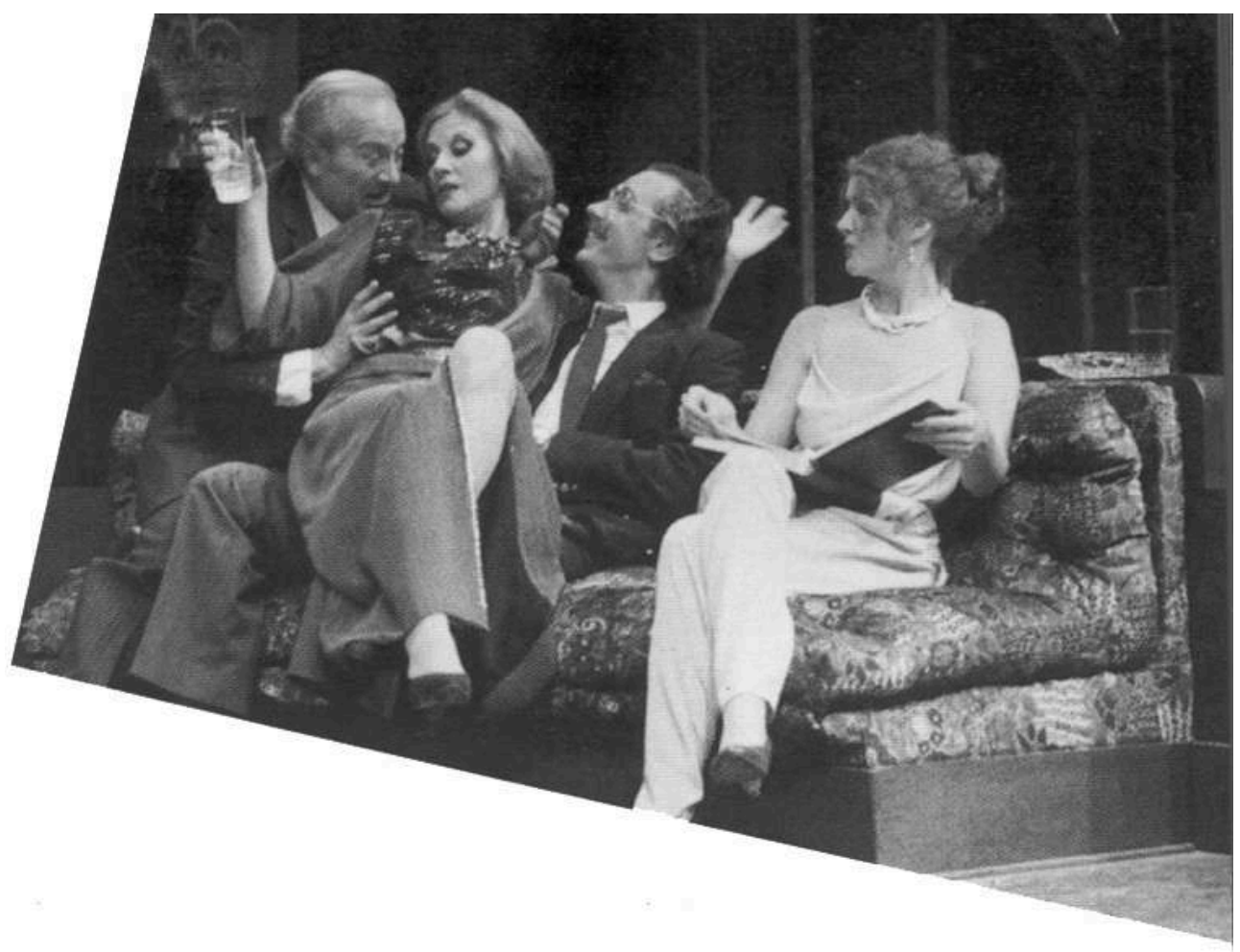

Cliché Zegers liberté faisait naître de nouvelles attentes. Mais l'impulsion donnée à la dramaturgie durant les années antérieures s'affaiblit. Selon Halpern, cité par María Teresa Zegers Nachbauer,

À quelques exceptions près, le théâtre a cessé d'attirer de nombreuses personnes qui avaient trouvé en lui, durant la dictature, un espace inégalable de rébellion. [...] Peut-être qu'après dix-sept ans d'autoritarisme, la transition de la dictature à la démocratie a laissé les gens de théâtre subitement dépourvus de ce qui avait été la matière première essentielle à la création dramaturgique : l'impact traumatisant de la répression d'un gouvernement de fait sur la société chilienne. ${ }^{8}$

Ceci dit, une nouvelle expression scénique surgit autour des années 90, avec des metteurs en scène comme Alfredo Castro, Ramón Griffero, Rodrigo Pérez. Sur le plan dramaturgique, Marco Antonio de la Parra et un nouveau venu, Benjamin Galemiri, consolident leur position. En 1992, quarante créations nationales voient le jour, contre seulement dix-huit en 1990. Les auteurs de la génération de 1950 occupent toujours le devant de la scène, puisque sont représentées Álamos en la azotea de Egon Wolff et El 
Guante de Hierro de Jorge Díaz. En 1993 a lieu au Chili le XXVIII ${ }^{\mathrm{e}}$ Festival mondial du théâtre des Nations. Cette rencontre a réuni 27 pays, 18 compagnies étrangères, 20 montages internationaux, 55 invités spéciaux, 36 compagnies nationales de la région métropolitaine, 48 œuvres nationales inscrites officiellement ${ }^{9}$.

Quant aux thèmes abordés par le théâtre, ils continuent à exprimer le scepticisme face au régime politique en place :

Le second gouvernement démocratique ne fait rien d'autre qu'approfondir le scepticisme et la distance que le théâtre assume face au système politique et économique régnant, et le style de vie qui se manifeste même en démocratie. Ce scepticisme et ce désenchantement que vivent les noyaux artistiques et intellectuels chilien s'expriment également dans le milieu théâtral. Ce sentiment commence à se faire sentir dans les œuvres présentées : un autre type de menace, de violence, de détresse matérielle et émotionnelle parcourent les scènes [... ${ }^{10}$

Il semblerait que le théâtre soit à nouveau entré en crise. Le public ne se précipite pas dans les salles, même si certaines œuvres jouissent d'un succès confortable. Mais si l'on en croit María Teresa Zegers Nachbauer, les crises à répétition feraient partie des caractéristiques du théâtre chilien :

Régulièrement, de façon cyclique, on parle de crise au théâtre. Ce fait, qui semble quelque peu récurrent dans la déjà longue histoire du théâtre, a été comparé à un malade chronique, auquel on a donné l'extrême-onction à de nombreuses reprises, mais qui refuse de mourir et qui, au lieu de respecter les prédictions de mauvaise augure qui annoncent son décès, se lève et marche avec une vitalité renouvelée.

Les pronostics pessimistes qui régulièrement se lèvent, se sont brisés contre un théâtre qui continue à survivre, s'adaptant au changement dans un monde de compétition. Ce que l'on appelle «crises»; indique des ajustements, une croissance, une transformation et une recherche. On a beau préparer les discours post-mortem, ceux que l'on prononce après le dernier coup de pelle, le théâtre au Chili et dans le monde n'agonise pas: il revit et ressuscite à chaque applaudissement, avec des va-et-vient, mais vivant pour les siècles des siècles. ${ }^{11}$

Même s'il peut apparaître comme le "pauvre » de la littérature au Chili, le théâtre est encore vigoureux et certains auteurs phares de la génération de 1950 continuent à produire des œuvres de qualité (nous pensons à Egon Wolff ou Jorge Díaz), et la relève semble assurée, notamment en la personne de Benjamin Galemiri.

\section{NOTES}

1. Déclaration de Fernando Debesa, citée dans mUNizAGa Giselle, HURTADo Maria de la Luz: Testimonios de teatro, 35 anos de teatro en la Universidad Católica, Santiago, Ed. Nueva Universidad, 1980 , p. 23.

2. Déclaration de Pedro Mortheiru; ibid, p. 29.

3. Déclaration de Fernando Debesa ; ibid, p. 31.

4. Ibid, p. 38.

5. HURTADo Maria de la Luz, ochsenius Carlos: Transformaciones del teatro chileno en la década del setenta, Santiago, Ceneca, 1982. 
6. La Balsa de la Medusa (Le Radeau de la Méduse) de Egon Wolff, nous invitons le lecteur à se reporter aux numéros 16 et 17 de Coulisses, comportant un dossier "spécial Radeau de la Méduse ", et au numéro 22 où figure une interview du dramaturge Chilien.

7. NACHBAUER ZEGERS Maria Teresa: 25 años de teatro, Santiago, departamento de programas culturales de la division de cultura del ministerio de Educación, 1999, p. 104.

8. Ibid, p. 122.

9. Ibid, p. 127.

10. Ibid, p. 128.

11. Ibid, p. 133.

\section{AUTEURS}

\section{CAROLE MICHEL}

Doctorante 